\title{
Inhaltsverzeichnis Band I
}

\section{Kapitel A}

\section{Einfïhrung}

1. Sozio-kulturelle Determinanten des Führungswandels . . . . . . . 1

2. Kooperative Führung als Teilaspekt der Humanisierung des Arbeitslebens .................. 3

3. Führung als interdisziplinäres Konzept . . . . . . . . . . 5

4. Zum Problemkomplex ,Kooperative Führung" . . . . . . . . 8

5. Ein integratives Bezugssystem zur Analyse von Führung in Orga-

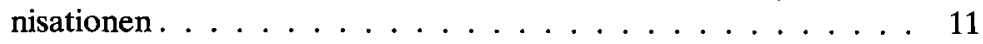

Ausgewählte Literatur . . . . . . . . . . . . . . . 12

\section{Kapitel B}

\section{Wissenschaftstheoretische und methodologische Grundlagen} der Führungsforschung

0. Zusammenfassung . . . . . . . . . . . . . . . 14

1. Wissenschaftstheoretische Aspekte der Führungsforschung . . . . 15

1.1. Führung als unabhängige, intervenierende und abhängige Variable 15

1.2. Analyseebenen . . . . . . . . . . . . . . . . . 19

1.2.1. Beschreibende Ebene . . . . . . . . . . . . . . . . . . . . . 19

1.2.2. Erklärende Ebene . . . . . . . . . . . . . . . . . 19

1.2.3. Spekulative Ebene . . . . . . . . . . . . . . . . 22

1.2.4. Wertende Ebene . . . . . . . . . . . . . . . 22

1.2.5. Pragmatische Ebene . . . . . . . . . . . . . . . . 26

1.3. Ein metatheoretisches Analysemodell der Führung . . . . . . . . 26

1.4. Atomistische vs. holistische Ansätze - zwei komplementäre Sichtweisen in der Führungsforschung . . . . . . . . . . . . . . 27

1.5. Definitionsarten sozialwissenschaftlicher Begriffe . . . . . . . . . 29

1.6. Struktur und Funktion sozialwissenschaftlicher Theorien . . . . . 32

1.7. Kriterienkatalog für die Beurteilung von Theorien in der Führungsforschung ....................... 34

2. Methodische Aspekte der Führungsforschung . . . . . . . . . 37

2.1. Das Experiment. . . . . . . . . . . . . . . . . . 37

2.2. Meßmethodische Gütekriterien. . . . . . . . . . . . . 39

2.2.1. Objektivität. . . . . . . . . . . . . . . . . . 39

2.2.2. Reliabilität (Verläßlichkeit) . . . . . . . . . . . . . . 39

2.2.3. Validität (Gültigkeit) . . . . . . . . . . . . . . . . . . 39 
2.3. Grundzüge der Korrelationsstatistik . . . . . . . . . . . . . . . 41

2.4. Grundzüge der Faktorenanalyse . . . . . . . . . . . . . . 46

Ausgewählte Literatur . . . . . . . . . . . . . . . . . . . . . . . 51

\section{Kapitel C}

\section{Begriffe über Führung}

0. Zusammenfassung ..................... 52

1. Probleme der Begriffsbestimmung von ,Führung" und „Führer" 52

1.1. Begriffsexplikation von ,Führung“. . . . . . . . . . . 54

1.2. Unterscheidung zwischen „Führung" und ,Führer" . . . . . . . 55

2. Grundlegende Charakterisierung von Führungsprozessen . . . . . 56

2.1. Merkmale der Führung . . . . . . . . . . . . . . . . . 56

2.2. Führungsdefinitionen . . . . . . . . . . . . . 61

3. Abgrenzung von verwandten Begriffen . . . . . . . . . . 63

3.1. Leitung . . . . . . . . . . . . . . . . . . . 63

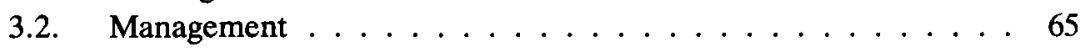

3.3. Macht . . . . . . . . . . . . . . . 65

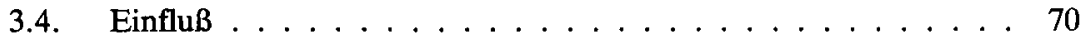

3.5. Herrschaft. . . . . . . . . . . . . . . . 71

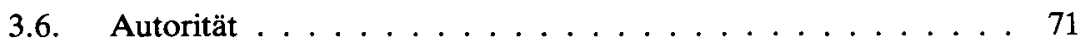

3.7. Vergleichende Darstellung der Begriffe . . . . . . . . . 73

Ausgewählte Literatur . . . . . . . . . . . . . . . . . . . 73

\section{Kapitel D}

\section{Theorien über den Menschen (Menschenbilder)}

0. Zusammenfassung . . . . . . . . . . . . . 75

1. Problemstellung und -abgrenzung . . . . . . . . . . 76

1.1. Menschenbilder als Problem der Wissenschaft und Praxis . . . . . 76

1.2. Die Bedeutung von Menschenbildern in der Führungsbeziehung. . 77

1.3. Menschenbilder im Umfeld der Führungsbeziehung . . . . . . . . 77

1.4. Von der Suche nach dem ,,richtigen" Führungsstil zur Situations-

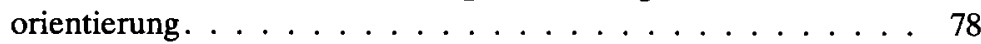

1.5. Menschenbild-Typologien . . . . . . . . . . . . . 80

2. Implizite Persönlichkeitstheorien . . . . . . . . . . . . . . . 84

3. Das Konzept des ,,Ökonomischen Menschen“ . . . . . . . . . . . 89

3.1. Sozialphilosophische Grundlagen. . . . . . . . . . . . . . . . . 89

3.2. Das Menschenbild des Taylorismus. . . . . . . . . . . . . . . 92

3.3. Erwartungen an Mitarbeiter ... . . . . . . . . . . . 92

3.4. Erwartungen an Vorgesetzte ............... 93

3.5. Die Beziehungen zwischen Mitarbeitern und Vorgesetzten . . . . 93 
3.6. Taylorismus und kooperative Führung . . . . . . . . . . . . . . 95

4. Das Konzept des ,,Sozialen Menschen“ . . . . . . . . . . . . 95

4.1. Die Human-Relations-Bewegung. . . . . . . . . . . . . . . . 95

4.2. Erwartungen an Mitarbeiter . . . . . . . . . . . . . . . 96

4.3. Erwartungen an Vorgesetzte . . . . . . . . . . . . . . 97

4.4. Bedeutung des Konzepts des ,,Sozialen Menschen“ für die kooperative Führung . . . . . . . . . . . . . . . . . . . 98

4.5. Exkurs: Der ,Organisationsmensch“. . . . . . . . . . . . 99

5. Das Konzept des nach Selbstentfaltung strebenden Menschen . . . 100

5.1. Die Suche nach ,,Human Resources“. . . . . . . . . . . . . . 100

5.2. Erwartungen an Mitarbeiter . . . . . . . . . . . . . . . . 102

5.3. Erwartungen an Vorgesetzte . . . . . . . . . . . . . . 103

5.4. Kooperative Führung im Modell des nach Selbstentfaltung strebenden Menschen. . . . . . . . . . . . . . . . . . 104

5.4.1. Führung oder Eigenmotivation? . . . . . . . . . . . . . . . 104

5.4.2. Überschätzung interpersonaler Beziehungen . . . . . . . . . . . 106

6. Das Konzept des ,,Komplexen Menschen“. . . . . . . . . . . . . 106

6.1. Erwartungen an Mitarbeiter . . . . . . . . . . . . . . 107

6.2. Erwartungen an Vorgesetzte . . . . . . . . . . . . . 107

6.3. Kooperative Führung und „Komplexer Mensch“ . . . . . . . . . 107

7. Das Menschenbild in der kooperativen Führung . . . . . . . . . . 109

Ausgewählte Literatur . . . . . . . . . . . . . . . . . . . 111

\section{Kapitel E}

\section{Theorien über die Führung}

0. Vorbemerkungen . . . . . . . . . . . . . . . . . . 112

1. Eigenschaftstheorie der Führung (Great-Man-Theorie) . . . . . 113

1.1. Korrelationsstatistische empirische Befunde (1904-1947) . . . . . 114

1.2. Korrelationsstatistische empirische Befunde (1948-1970) . . . . . 117

1.3. Empirische Beziehungen zwischen Führungseigenschaften und Gruppenerfolg . . . . . . . . . . . . . . . . 119

1.4. Bewertung der Eigenschaftstheorie der Führung . . . . . . . . . 122

1.4.1. Zur Problematik des Eigenschafts-Konzeptes . . . . . . . . . 123

1.4.2. Konsistenz . . . . . . . . . . . . . . . . . . . . . . . 125

1.4.3. Generalität-Spezifität (Intraindividuelle Dimension). . . . . . . . 125

1.4.4. Universalität-Singularität (Interindividuelle Dimension) . . . . . 125

1.4.5. Zusammenfassende Bewertung der Eigenschafts-Theorie der Führung . . . . . . . . . . . . . . . . . 127

2. Rollentheorie der Führung . . . . . . . . . . . . . . . . 129

2.1. Dimensionen des Rollenkonzeptes . . . . . . . . . . . . . 133

2.2. Bewertung . . . . . . . . . . . . . . . . . 133

3. Situationstheorie der Führung . . . . . . . . . . . 134 
3.1. Grundlagen . . . . . . . . . . . . . . . . . . . . 134

3.2. Bewertung . . . . . . . . . . . . . . . . 135

4. Eine neuere Motivationstheorie der Führung: Die Weg-Ziel-

Theorie . . . . . . . . . . . . . . . . . . . 136

4.1. Grundlagen . . . . . . . . . . . . . . . . 136

4.2. Bewertung . . . . . . . . . . . . . . . . 140

5. Verhaltenstheorie der Führung . . . . . . . . . . . . . . . 141

5.1. Führungsfunktionen . . . . . . . . . . . . . . 141

5.2. Bewertung des Konzepts der Führungsfunktionen . . . . . . . . 142

5.3. Die Verhaltenstheorie der Führung von Yukl . . . . . . . . . 143

5.3.1. Das Diskrepanz-Modell. . . . . . . . . . . . . . . . . . . . . . 143

5.3.2. Das Multiple-Verbindungs-Modell . . . . . . . . . . . . . . 144

5.4. Bewertung der Verhaltenstheorie der Führung von Yukl. . . . . . 145

6. Interaktionstheorie der Führung . . . . . . . . . . . . . . 145

6.1. Grundbegriffe der Austauschtheorie . . . . . . . . . . . . . 146

6.2. Grundbegriffe der Gleichheits-Theorie (Equity-Theorie) . . . . 148

6.3. Bedingungen sozialer Interaktion. . . . . . . . . . . . . . . . 151

6.4. Formen sozialer Interaktion . . . . . . . . . . . . . . 155

6.5. Bewertung . . . . . . . . . . . . . . . . . 160

7. Psychoanalytische Beiträge zur Führung . . . . . . . . . 160

7.1. Die psychoanalytische Sichtweise . . . . . . . . . . . . . 161

7.2. Die psychoanalytische ,Führungstheorie“ . . . . . . . . . 161

7.2.1. Der Begriff ,Identifizierung“. . . . . . . . . . . . . . . 162

7.2.2. Identifizierung in der Führungsbeziehung . . . . . . . . . 162

7.2.3. Übertragung . . . . . . . . . . . . . . . . . . . . . . 162

7.2.4. Identifikation mit dem ,,Aggressor" . . . . . . . . . . . 163

7.2.5. Die Gruppenbildung um eine zentrale Person . . . . . . . . . . 163

7.2.6. Das Konzept der Gesamtgruppe . . . . . . . . . . . . . . . . 164

7.3. Bewertung . . . . . . . . . . . . . . . . . . . . 164

8. Wissenschaftstheoretische Bewertung der Führungstheorien. . . . 164

Ausgewählte Literatur . . . . . . . . . . . . . . . . . 166

\section{Kapitel F}

\section{Theorien über das Mitarbeiterverhalten (Motivationstheorien)}

0. Zusammenfassung . . . . . . . . . . . . . . 168

1. Zur Arbeitsmotivation in der Managementlehre . . . . . . . . . 168

1.1. Zum Motivationsbegriff. . . . . . . . . . . . . . . . . . . . 169

1.2. Arbeitsmotivation . . . . . . . . . . . . . . . . . 171

1.3. Das Motivationskonzept der „Wissenschaftlichen Betriebsführung" . . . . . . . . . . . . . . . . . . . 172

1.4. Das Motivationskonzept des Human-Relations-Ansatzes . . . . . 173

2. Motivationskonzepte des Human-Resources-Modells . . . . . . 174 
2.1. Die Motivationstheorie Maslows . . . . . . . . . . . . . . . 176

2.1.1. Bewertung der Bedürfnishierarchie. . . . . . . . . . . . 178

2.1.2. Empirische Überprüfungsversuche der Bedürfnishierarchie . . . . 180

2.1.3. Folgerungen aus der Bedürfnishierarchie für die Führung . . . . . 185

2.2. Die E.R.G.-Theorie von Alderfer - Eine modifizierte Version der Maslowschen Bedürfnishierarchie . . . . . . . . . . . 186

2.3. Die Zwei-Faktoren-Theorie der Arbeitsmotivation von Herzberg . . . . . . . . . . . . . . . . . 188

2.3.1. Präzisierungs-Versuche der Zwei-Faktoren-Theorie . . . . . . . . 191

2.3.2. Folgerungen aus der Zwei-Faktoren-Theorie für die Führung . . . 191

2.3.3. Neuere Entwicklungstendenzen der Zwei-Faktoren-Theorie und ihre Bedeutung für die Führungspraxis . . . . . . . . . . . . . 192

2.3.4. Bewertung der Zwei-Faktoren-Theorie. . . . . . . . . . . . . . . 194

3. Neuere kognitive Motivationstheorien in der Managementlehre und Organisationspsychologie - Theorien der Leistungsmotivation . . . . . . . . . . . . . . . . . . 195

3.1. Das kognitive Erwartungs-Wert-Modell von Vroom . . . . . . . . 196

3.2. Das kognitive Erwartungs-Wert-Modell von Lawler . . . . . . . . 199

3.3. Das integrierte Erwartungs-Wert-Modell von Porter/Lawler . . . . 201

3.4. Bewertung der Erwartungs-Wert-Theorien. . . . . . . . . . . . . 204

3.5. Die Theorie der Leistungsmotivation von Atkinson . . . . . . . . 206

3.5.1. Begriffsklärung . . . . . . . . . . . . . . . . 206

3.5.2. Die theoretische Aussage . . . . . . . . . . . . . . . . 206

3.6. Das erweiterte Modell der Leistungsmotivation von Heckhausen 210

3.7. Leistungsmotivation und Attribuierungstheorie . . . . . . . . . 212

3.7.1. Formen der Ursachenzuschreibung . . . . . . . . . . . . . . 212

3.7.2. Bedingungen der Ursachenzuschreibung . . . . . . . . . . . . . 213

3.8. Attribuierung, Vertrauen und Führung. . . . . . . . . . . . . . . 216

Ausgewählte Literatur . . . . . . . . . . . . . . . . . . . . . . 217

\section{Kapitel G}

\section{Verhaltensmuster der Führung (Führungsstile)}

0. Zusammenfassung . . . . . . . . . . . . . . . . 218

1. Begriffsbestimmung von ,Führungsstil“ . . . . . . . . . . 220

2. Die klassische Führungsstiltypologie von Lewin: autokratisch, demokratisch, laissez-faire . . . . . . . . . . . . . 222

2.1. Darstellung der Führungsstiltypologie . . . . . . . . . . . 222

2.2. Diskussion . . . . . . . . . . . . . . . . . 223

3. Idealtypische Konzepte des Führungsverhaltens . . . . . . . . . 225

3.1. Eindimensionale Ansätze . . . . . . . . . . . . . . . . . . . . 225

3.1.1. Der entscheidungsorientierte Ansatz von Tannenbaum/Schmidt . . 225

3.1.2. Diskussion . . . . . . . . . . . . . . . . . . . 225 
3.2. Zweidimensionale Ansätze . . . . . . . . . . . . . . . 225

3.2.1. Das Verhaltensgitter (Managerial Grid) von Blake/Mouton . . . . 225

3.2.2. Diskussion . . . . . . . . . . . . . . . . . . . 227

3.3. Dreidimensionale Ansätze . . . . . . . . . . . . . . . . . 231

3.3.1. Die 3-D-Theorie der Führung von Reddin . . . . . . . . . . . 231

3.3.2. Diskussion . . . . . . . . . . . . . . . . . . . 232

3.3.3. Die situative ,Reifegrad“-Theorie von Hersey und Blanchard. . . 232

3.3.4. Diskussion . . . . . . . . . . . . . . . . . 233

3.4. Der vierdimensionale Ansatz von Farris . . . . . . . . . . . . 234

3.5. Vieldimensionale Ansätze . . . . . . . . . . . . . . 235

3.5.1. Das System organisatorischer Führungselemente von Bleicher. . . 235

3.5.2. Das System organisatorischer und personaler Führungselemente von Lattmann . . . . . . . . . . . . . . . . . . 237

3.5.3. Diskussion . . . . . . . . . . . . . . . . . . . . 238

4. Empirisch ermittelte Konzepte des Führungsverhaltens . . . . . . 238

4.1. Die Ohio-Studien zum Führungsverhalten . . . . . . . . . . . 239

4.1.1. Consideration (C) und Initiating Structure (I) . . . . . . . . 242

4.1.2. Diskussion . . . . . . . . . . . . . . . . . . 247

4.1.3. Psychometrische Beurteilung der „,Ohio State Leadership Scales“ 248

4.1.4. Zum gegenwärtigen Stand der C- und I-Forschung . . . . . . . . . 249

4.2. Die Michigan-Studien zum Führungsverhalten . . . . . . . . . . . 256

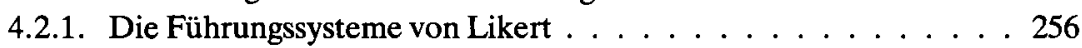

4.2.2. Diskussion . . . . . . . . . . . . . . . . . 260

4.3. Das Typenkonzept von Tausch . . . . . . . . . . . . . . . 260

4.4. Das Kontingenzmodell der effektiven Führung von Fiedler . . . . 261

4.4.1. Die drei Situationsvariablen . . . . . . . . . . . . . . . 262

4.4.2. „Günstigkeit“ der Situation . . . . . . . . . . . . . . 265

4.4.3. Diskussion . . . . . . . . . . . . . . . 266

4.4.4. Folgerungen für die Praxis . . . . . . . . . . . . . . 270

4.5. Ein neuer dimensionaler Ansatz in der Führungsstilforschung . . 270

5. Allgemeine Bewertung der Führungsstiltypologien. . . . . . . . 272

5.1. Ziele und Bedingungen von Führungsstilklassifikationen . . . . . 272

5.2. Folgerungen für die Praxis . . . . . . . . . . . . . . . . . . . 274

Ausgewählte Literatur . . . . . . . . . . . . . . . . . . . . 274

\section{Kapitel H}

\section{Gestaltungsmuster der Führung (Managementkonzeptionen)}

0. Zusammenfassung . . . . . . . . . . . . . . 275

1. Managementtheorien . . . . . . . . . . . . . . 276

2. Historische Entwicklung der Managementlehre . . . . . . . . . 277

2.1. Periode I - Vorwissenschaftliches Management . . . . . . . . . . 278

2.2. Periode II - ,Wissenschaftliche Betriebsführung“, bürokratisches Modell und Theorie des administrativen Managements . . . 278 
2.3. Periode III - ,Human-Relations-Modell“ . . . . . . . . . . . . 282

2.4. Periode IV - Differenzierung und Weiterentwicklung der Managementlehre. . . . . . . . . . . . . . . . 283

3. Führungsmodelle für Gesamtsysteme - ,Management-by-Ansätze“ . . . . . . . . . . . . . . . . . 285

3.1. Management by Ideas. . . . . . . . . . . . . . . . . . . 287

3.1.1. Darstellung . . . . . . . . . . . . . . . . . . . . . . . 287

3.1.2. Bedeutung für die Führung . . . . . . . . . . . . . . . . 287

3.2. Management by Break Through . . . . . . . . . . . . . 287

3.2.1. Darstellung . . . . . . . . . . . . . . . . . . . 287

3.2.2. Bedeutung für die Führung . . . . . . . . . . . . . . . 288

3.3. Management by Delegation. . . . . . . . . . . . . . . . . 288

3.3.1. Das Harzburger Modell (HM) . . . . . . . . . . . . . . 288

3.3.2. Beurteilung des HM . . . . . . . . . . . . . . . . . . 290

3.3.3. Bedeutung für die Führung . . . . . . . . . . . . . . . . . 292

3.4. Management by Objectives (MBO). . . . . . . . . . . . . 305

3.4.1. Darstellung . . . . . . . . . . . . . . . . . . 306

3.4.2. Bedeutung für die Führung . . . . . . . . . . . . . . . . . . 309

3.5. Kritische Beurteilung von Managementprinzipien . . . . . . . 310

Ausgewählte Literatur . . . . . . . . . . . . . . . . . . . . . . 311

\section{Kapitel I}

\section{Gestaltungsmuster sozio-technischer Systeme (Organisationsstrukturen)}

0. Zusammenfassung . . . . . . . . . . . . . . . . . . . 312

1. Problemstellung und Begriffsklärung . . . . . . . . . . . . . 312

1.1. Die bürokratische Organisation als Umfeld der Führung . . . . . . 312

1.2. Bürokratie und demokratische Gesellschaft . . . . . . . . . . . . 313

1.3. Zum Begriff der Organisation und der Bürokratie . . . . . . . . . 315

2. Klassische Bürokratiemodelle . . . . . . . . . . . . . . . 316

2.1. Die Entstehung bürokratischer Verwaltungsformen . . . . . . . . 316

2.2. Das Bürokratiemodell Max Webers . . . . . . . . . . . . . . . 318

2.2.1. Merkmale der Bürokratie . . . . . . . . . . . . . . . 318

2.2.2. Drei Typen der Herrschaft . . . . . . . . . . . . . . . . . . . 320

2.2.3. Das idealtypische Mitglied von Bürokratien (,Beamter" ${ }^{4}$. . . . . 320

2.3. Bürokratietypen . . . . . . . . . . . . . . . . . . 322

2.3.1. Die Strukturalisten . . . . . . . . . . . . . . . . . . 322

2.3.2. Drei Modelle der Bürokratie . . . . . . . . . . . . . . . . . . 323

2.4. Hierarchische Strukturformen der Organisationstheorie . . . . . 327

2.4.1. Strukturmerkmale . . . . . . . . . . . . . . . 327

2.4.2. Organisatorische Strukturtypen. . . . . . . . . . . . . . . 329

2.5. Zur Kritik bürokratischer Modelle . . . . . . . . . . . . . . . . . 334

2.5.1. Vom Bürokratie- zum ,Mülleimer"-Modell . . . . . . . . . . . . 334 
2.5.2. Gründe für die Institutionalisierung bürokratischer Strukturen . . 341

2.5.3. Zur Kritik bürokratischer Institutionen. . . . . . . . . . . . . 342

3. Das Organisationskonzept der Aston-Gruppe . . . . . . . . . . 344

3.1. Grundlagen des Aston-Modells . . . . . . . . . . . . . . . . . . . 344

3.1.1. Grundannahmen . . . . . . . . . . . . . . . . . . . . 345

3.1.2. Das Variablensystem . . . . . . . . . . . . . . . . . . . . 345

3.2. Die Rollentheorie als zentrales Paradigma des Aston-Modells . . . 347

3.2.1. Zur Bedeutung der Rollentheorie . . . . . . . . . . . . . . 347

3.2.2. Anforderungen der kooperativen Führung an die Rollentheorie . . 348

3.2.3. Einige Konzepte der Rollentheorie . . . . . . . . . . . . . . . . . 349

3.3. Korrelative Beziehungen zwischen Variablen soziotechnischer Systeme . . . . . . . . . . . . . . . . . . 352

3.4. Der Einfluß der Organisationsstruktur auf Rollenerwartungen und Rollenhandeln . . . . . . . . . . . . . . 356

3.4.1. Situative Gestaltung des Rollenhandelns . . . . . . . . . . . . 356

3.4.2. Zur Messung von Verhaltensspielräumen . . . . . . . . . . . 358

3.5. Das Modell der organisatorischen Steuerung individuellen Verhaltens . . . . . . . . . . . . . . . . . . 360

3.6. Ergebnisse der Aston-Studien . . . . . . . . . . . . . 362

3.7. Kritik des Aston-Ansatzes . . . . . . . . . . . . . . . . 365

3.7.1. Begriffe und Grundannahmen . . . . . . . . . . . . . . 365

3.7.2. Zum Problem der Kausalbeziehung. . . . . . . . . . . . . 366

4. Organisationsumgebung . . . . . . . . . . . . . 368

4.1. Der Umwelteinfluß auf einzelne Führungsbereiche . . . . . . . 368

4.2. Begriffe und Paradigmata der Umgebung . . . . . . . . . . . . . 371

4.3. Umfang und Gestalt der Organisationsumgebung . . . . . . . . . 375

4.3.1. ,Umgebung“ im Aston-Modell. . . . . . . . . . . . . . . . . . . 375

4.3.2. Vergleich einiger Begriffsbestimmungen von ,Umgebung“ . . . . 376

4.3.3. Versuch einer generellen Bestimmung von ,Umgebung““ . . . . . 378

4.4. Problematisierung der Umgebungsgrenze . . . . . . . . . . . . 379

4.5. Sektoren und Komponenten der Umgebung . . . . . . . . . . . 381

4.6. Qualitative Merkmale der Umgebung . . . . . . . . . . . . . . . 383

4.7. Abhängigkeiten zwischen Organisation, Umgebung und Führungsbereich . . . . . . . . . . . . . . . 384

4.8. Zur Organisation der Austauschbeziehungen zwischen System und Umgebung . . . . . . . . . . . . . . . . . . . . 388

5. Politisch-gesellschaftliche Normen als Variable der Organisationsumgebung. . . . . . . . . . . . . . . . . . . 390

5.1. Rechtlich-politischer Einfluß auf die Organisationsstruktur . . . . 390

5.2. Mitbestimmungsgesetz . . . . . . . . . . . . . . . . . . 394

5.3. Betriebsverfassungsgesetz . . . . . . . . . . . . . 395

5.4. Personalvertretungsgesetze . . . . . . . . . . . . . . 401

6. Organisationsspezifische Normen. . . . . . . . . . . . . 403

6.1. Die Bedeutung organisationsinterner Normensysteme . . . . . . . 403

6.1.1. Zusammenhänge zwischen verschiedenen Normensystemen . . . . 403 
6.1.2. Zur Unterscheidung von Organisationsgrundsätzen und Verhaltensleitsätzen . . . . . . . . . . . . . . . . . . . 404

6.2. Organisationsgrundsätze - Unternehmensgrundsätze . . . . . . 405

6.2.1. Personale und soziale Aspekte . . . . . . . . . . . . . . . . 405

6.2.2. Darstellung von Organisationszielen . . . . . . . . . . . . . 407

6.2.3. Organisationsgrundsätze und Humanisierung der Arbeit. . . . . . 409

6.3. Organisationsprinzipien - Ein Beispiel: Die Gemeinsame Geschäftsordnung der Bundesministerien (GGO) . . . . . . . . . . . 419

6.3.1. GGO und kooperative Führung . . . . . . . . . . . . . . . . . 419

6.3.2. Zusammenfassende Stellungnahme zur Ergänzung der GGO . . . 424

6.4. Verhaltensleitsätze . . . . . . . . . . . . . . . . . . . . 424

6.4.1. Zur Begriffsklärung. . . . . . . . . . . . . . . . . . . . . . 424

6.4.2. Funktion von Verhaltensleitsätzen . . . . . . . . . . . . . 426

6.4.3. Inhalte von Verhaltensleitsätzen . . . . . . . . . . . . . . . 428

6.4.4. Verhaltensleitsätze als Instrument rationaler Kommunikation . . . 432

6.5. Zur Einführung von Organisationsgrundsätzen und Verhaltensleitsätzen . . . . . . . . . . . . . . . 433

6.6. Die Formalisierung organisatorischer Normen . . . . . . . . . . . 438

6.7. Organisationsziele und Verhaltensleitsätze . . . . . . . . . . . . 439

Ausgewählte Literatur . . . . . . . . . . . . . . . . . . . . . . . . . 440

Anhang $(\mathrm{I}-\mathrm{IV}) \ldots \ldots \ldots \ldots \ldots \ldots \ldots \ldots$

Literaturverzeichnis . . . . . . . . . . . . . . . . . . . 467

Namenregister . . . . . . . . . . . . . . . . 513

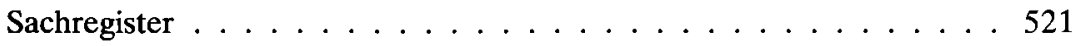


